\title{
Inflammatory Mediator Profiling Reveals Immune Properties of Chemotactic Gradients and Macrophage Mediator Production Inhibition during Thioglycollate Elicited Peritoneal Inflammation
}

\author{
Derek Lam, Devon Harris, and Zhenyu Qin \\ Division of Vascular Surgery, Department of Surgery, University of Texas Health Science Center at San Antonio, \\ San Antonio, TX 78229, USA \\ Correspondence should be addressed to Zhenyu Qin; qinz@uthscsa.edu
}

Received 28 December 2012; Revised 17 February 2013; Accepted 24 February 2013

Academic Editor: Miao Wang

Copyright ( 2013 Derek Lam et al. This is an open access article distributed under the Creative Commons Attribution License, which permits unrestricted use, distribution, and reproduction in any medium, provided the original work is properly cited.

\begin{abstract}
Understanding of spatiotemporal profiling of inflammatory mediators and their associations with $\mathrm{M} \Phi$ accumulation is crucial to elucidate the complex immune properties. Here, we used murine thioglycollate elicited peritonitis to determine concentrations of 23 inflammatory mediators in peritoneal exudates and plasma before (day 0 ) and after (days 1 and 3 ) thioglycollate administration to peritoneal cavities; these mediators included TNF- $\alpha$, FGF-9, IFN- $\gamma$, IP-10, RANTES, IL-1 $\alpha$, IL-6, IL-7, IL-10, IL-11, IL-12p70, IL17A, lymphotactin, OSM, KC/GRO, SCF, MIP-1 $\beta$, MIP-2, TIMP-1, VEGF-A, MCP-1, MCP-3, and MCP-5. Our results showed that concentrations of most mediators in exudates and plasma reached peak levels on day 1 and were significantly reduced on day 3 . Conversely, $\mathrm{M} \Phi$ numbers started to increase on day 1 and reached peak levels on day 3 . Moreover, LPS treatment in vitro significantly induced mediator productions in cell culture media and lysates from $М \Phi$ isolated on day 3. Our results also showed that on day 0, concentrations of many mediators in plasma were higher than those in exudates, whereas on day 1, the trend was reversed. Overall, the findings from thioglycollate elicited peritonitis reveal that reversible chemotactic gradients between peritoneal exudates and blood exist in basal and inflamed conditions and the inflammatory mediator production in vivo is disassociated with macrophage accumulation during inflammation resolution.
\end{abstract}

\section{Introduction}

During the early stage of inflammatory diseases, the gradients of some of the inflammatory mediators play important roles in the recruitment of leukocytes to inflamed areas. In this stage, monocytes and macrophages $(M \Phi)$ are the secondary line of inflammatory cells after neutrophils. In contrast to the recruited neutrophils with short life spans due to apoptosis [1], МФ have longer life spans and play a more important role in the clearance of neutrophils via phagocytosis. In addition to leukocyte recruitment, another primary response to inflammation is the secretion of the inflammatory mediators, including both proinflammatory mediators such as interleukin (IL)-1, IL-6, and tumor necrosis factor (TNF)- $\alpha$, and anti-inflammatory mediators such as IL-10. These mediators are produced mainly by leukocytes within the inflamed areas and they elicit particular immune responses to target cells or tissues via specific receptors and signaling pathways. Thus, it is important to understand the spatiotemporal association between the inflammatory mediator profiling and leukocyte accumulation.

Murine thioglycollate elicited peritonitis is an appropriate model with which to study inflammatory events that are accompanied by inflammatory mediator production and leukocyte accumulation. In this model, neutrophil numbers start to increase and reach peak levels between 4 and 24 hours after treatment, while $M \Phi$ numbers start to increase at 24 hours and reach peak levels at 3 to 4 days. We reported that copper is accumulated in the peritoneal $\mathrm{M} \Phi$ lack of ATP7A [2]. The peritoneal cavity is colonized with leukocytes. Under steady conditions, the resident peritoneal cells include MФ, T cells, B cells, NK cells, mast cells, and 
dendritic cells $[3,4]$. Resident $M \Phi$ and dendritic cells provide a basal immune surveillance. Notably, a newly described population in adipose tissue, associated lymphoid clusters, has been observed in the murine peritoneal cavity [5]. In addition to these immune cells, mesothelial cells that line the peritoneum also serve as a source of inflammatory mediators within the peritoneal cavity [6,7]. Although controversy exists on the subject, the role of resident peritoneal $\mathrm{M} \Phi$ in thioglycollate elicited murine peritonitis appears to be minimal. For example, following the selective depletion of resident $М \Phi ~(86 \%$ reduction) by pretreatment of mice with liposomes containing $\mathrm{Cl}_{2} \mathrm{MDP}$ (clodronate), thioglycollate administration did not alter neutrophil accumulation into peritoneal cavities [8]. A similar finding also occurred when mast cells were selectively depleted ( $95 \%$ reduction) by the pretreatment of mice with compound 48/80 [8]. Interestingly, resident $\mathrm{M} \Phi$ depletion inhibited neutrophil influx in lipopolysaccharide (LPS) induced peritonitis, whereas MФ depletion increased neutrophil influx in zymosan induced peritonitis [8]. Previously, studies also showed that thioglycollate treatment ousted nearly all of the resident peritoneal MФ in the draining lymph nodes within 4 hours $[9,10]$. Although large-scale studies have been performed to investigate the association between thioglycollate induced inflammatory mediator production and peritoneal leukocyte accumulation in vivo, these studies were usually designed to elucidate elegant mechanisms that were related to the functions of specific proteins in these events. Therefore, a global view is needed of the inflammatory mediator profiling and its association with leukocyte accumulation. Thus, we recently investigated the concentrations of multiple inflammatory mediators in peritoneal exudates and plasma before (day 0 ) and after (day 1 and 3 ) thioglycollate administration to murine peritoneal cavities. This study reveals important immune properties that are related to chemotactic gradients and the inhibition of $\mathrm{M} \Phi$ mediator production in vivo.

\section{Materials and Methods}

2.1. Mice. C57BL/6 males (The Jackson Laboratory, Bar Harbor, ME, USA) were used for this study at 10-14 weeks old. The animal protocol was approved by Institutional Animal Care and Use Committee of the University of Texas Health Science Center at San Antonio.

2.2. Thioglycollate Induced Peritonitis. Peritoneal cavities were lavaged with $3 \%$ Brewer thioglycollate broth (SigmaAldrich, St. Louis, MO) to establish acute sterile peritonitis. During the peritoneal exudate collection, mice were excluded from further studies if obvious adhesions were observed between the peritoneum and skin and/or if marked increases were observed in peritoneal thickness of peritoneum.

2.3. Collection of Blood and Peritoneal Exudates. Mice were euthanized at indicated times, and heparinized blood was collected from the hearts. Blood samples were centrifuged at $250 \times \mathrm{g}$ for 5 minutes for plasma isolation. Subsequently, in order to collect peritoneal exudates, one milliliter of 1x phosphate buffered saline (PBS) was administered via intraperitoneal (i.p.) injection. Peritoneal exudates were harvested following 60 seconds of peritoneal massage. After centrifugation at $250 \times \mathrm{g}$ for 5 minutes, the supernatant exudates were collected. Both the plasma and exudate samples were stored at $-80^{\circ} \mathrm{C}$ before further studies. After the first administration of $1 \mathrm{~mL}$ of PBS, mice were administered a second dose of $3 \mathrm{~mL}$ of 1xPBS. The cell pellets from these two administrations were combined and resuspended in $1 \mathrm{~mL}$ of red blood cell lysis buffer (Hybri-Max, Sigma-Aldrich) at room temperature for $1 \mathrm{~min}$. Next, the mixtures were washed with RPMI 1640 medium (Hyclone, Logan, UT) that was supplemented with $10 \%$ fetal bovine serum (FBS), 100 units/mL of penicillin, and $100 \mathrm{mg} / \mathrm{L}$ of streptomycin. Cells were then pelleted and resuspended in the same growth medium at room temperature for further studies.

2.4. Flow Cytometry. Peritoneal exudate cells were first incubated with Fc block (anti-CD16/CD32) (BD Biosciences, San Jose, CA) for $20 \mathrm{~min}$ and stained for surface expression using anti-Ly6G-PE (BD Biosciences) and/or anti-F4/80-AF647 (Serotec, Oxford, UK) for $30 \mathrm{~min}$. All washing and staining were performed with $2 \%$ FBS in PBS. At least 22,000 cells were analyzed for each sample. The numbers of neutrophils and macrophages were calculated by multiplying the total cell number by the percentage of positive cells for each immunostain.

2.5. Peritoneal MФ Isolation and Treatment with Lipopolysaccharide (LPS). Peritoneal exudate cells were placed on polystyrene Petri dishes $(95 \mathrm{~mm} \times 15 \mathrm{~mm})$ at $1-0.5 \times$ $10^{6}$ cells $/ \mathrm{mL}$ for two hours at $37^{\circ} \mathrm{C}$ in a humidified $95 \%$ air$5 \% \mathrm{CO}_{2}$ atmosphere. Then these cells were washed twice to remove nonadherent cells. The $\mathrm{M} \Phi$ purity was confirmed by F4/80 staining and morphology.

LPS (Sigma-Aldrich) is extracted from Escherichia coli serotype O55:B5 and purified by gel filtration chromatography. LPS stock solution was prepared in double distilled water at a concentration of $5 \mathrm{mg} / \mathrm{mL}$. During the experiment, murine peritoneal $\mathrm{M} \Phi$ at $0.5 \times 10^{6}$ cells $/ \mathrm{mL}$ were first placed into a 24-well plate with growth area at $2 \mathrm{~cm}^{2}$ for $4 \mathrm{~h}$. Cells were then treated $24 \mathrm{~h}$ with LPS. After collection of cell culture media, the cell pellets were lysed with $50 \mathrm{mM}$ Tris$\mathrm{HCl}$ with $2 \mathrm{mM}$ EDTA, pH 7.4. Both cell culture media and lysates were stored at $-80^{\circ} \mathrm{C}$ before further studies.

2.6. Detection of Inflammatory Mediators. Twenty-three inflammatory mediators were analyzed in plasma, peritoneal exudates, cell culture media, and lysates using a bead-based multiplexing immunoassay (Myriad RBM, Austin, TX) [11, 12]. These mediators included $\operatorname{TNF}-\alpha$, fibroblast growth factor (FGF) 9, interferon (IFN) $\gamma$, IFN- $\gamma$-inducible protein (IP) 10, RANTES, IL-17A, IL-12p70, IL-11, IL-10, IL-7, IL-6, IL-1 $\alpha$, lymphotactin, oncostatin-M (OSM), growth-regulated $\alpha$ protein (KC/GRO), stem cell factor (SCF), macrophage inflammatory protein (MIP)-1 $\beta$, MIP-2, tissue inhibitor of metalloproteinase (TIMP)-1, vascular endothelial growth factor (VEGF)-A, monocyte chemotactic protein (MCP)-1, 
MCP-3, and MCP-5. For the analyses, samples were first thawed, vortexed, and centrifuged before being loaded into a 96-well microtiter plate. Next, the microtiter plate was placed onto a liquid handler machine in which the samples were automatically added to reaction wells that contained capture beads. The beads were incubated with the samples for 1 hour at room temperature to allow the antigens of interest to bind to their targets. Multiplexed cocktails of biotinylated, reporter antibodies for each multiplex were added to the bead mixtures, and were incubated for 1 hour at room temperature. Multiplexes were developed using an excess of streptavidinphycoerythrin solution that was thoroughly mixed into each multiplex and incubated for 1 hour at room temperature. The plates were washed to remove unbound detection reagents and were read on a Luminex 100 instrument, in which the excitation beams detected the fluorescent signals of each bead. A minimum of 50 beads were detected per protein, per sample. All values were reported as the means. For each multiplex, both calibrators and controls were included on each microtiter plate. This assay was validated by ELISA $[13,14]$ and has been widely used to determine murine inflammatory mediator profiling at the protein levels [15].

2.7. Statistics. For each inflammatory mediator, the mean \pm standard error (SE) was calculated for each experimental group. Data were compared by Student's $t$-test (two tails). Significant differences were defined as having $P$ values < 0.05 . For inflammatory mediator detection in plasma and exudates, if more than $50 \%$ of the samples from an experimental group were below the detection limit, the group was marked as undetectable. If fewer than (or equal to) $50 \%$ of samples from an experimental group were below the detection limit, the least detectable dose was used as the concentration of these samples for further statistics. For the fold increase determination in the LPS treatment studies, if the pretreatment sample values were below the detection limit, the least detectable dose was used to estimate the fold increase.

\section{Results}

In our studies, we first determined the total cell numbers and numbers of neutrophils and $M \Phi$ in the peritoneal exudates before and after thioglycollate treatment. As shown in Figure 1, the total numbers of cells in the peritoneal exudates increased nearly 10 -fold on day $1\left(10.74 \pm 0.54 \times 10^{6}\right.$ neutrophils and $5.48 \pm 0.28 \times 10^{6} \mathrm{M} \Phi ; n=3$ ) compared to day $0\left(0.87 \pm 0.06 \times 10^{4}\right.$ neutrophils and $1.07 \pm 0.07 \times 10^{6} \mathrm{M} \Phi$; $n=4)$, and reached peak levels on day $3\left(1.94 \pm 0.09 \times 10^{6}\right.$ neutrophils and $\left.40.19 \pm 1.87 \times 10^{6} \mathrm{M} \Phi ; n=4\right)$.

\subsection{Inflammatory Mediator Concentrations in Peritoneal Exu-} dates Reached the Peak on Day 1 Following Thioglycollate Treatment In Vivo. We analyzed the concentrations of 23 inflammatory mediators at three timepoints in peritoneal exudates following thioglycollate treatment. As shown in Figure 2, the concentrations of all mediators reached peak levels on day 1 . Based on the concentration levels in the

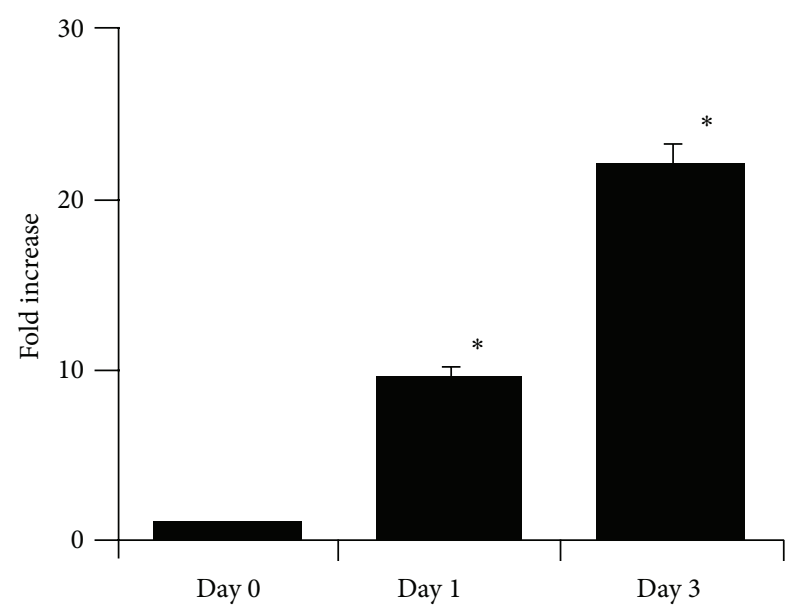

FIGURE 1: Fold increases of total cell numbers in peritoneal exudate during thioglycollate treatment in vivo. C57BL/6 mice (males) were treated with thioglycollate as described in Section 2. At three timepoints, before (day 0) and after (day 1 and 3) the treatment, peritoneal exudate cells were isolated and counted as described in Section 2. Fold increases were calculated as the cell number at a timepoint divided by the cell number on day 0 (defined as 1 ). Values are presented as the means \pm SE. $n=3-4$ per timepoint. ${ }^{*} P<0.001$ versus day 0 .

exudates on day 1 (Table 1 ), these mediators were divided into three groups. The concentrations of the first group, which included MCP-1, MCP-3, FGF-9, MIP-1 $\beta$, and TIMP1 , reached nanogram levels. Considering that these mediator concentrations were higher than those of other groups, these are likely the most important candidates to affect the induction of the $\mathrm{M} \Phi$ accumulation on day 3. The concentrations of the mediators in the second group were determined to be in the picogram range, including IL-17A, KC/GRO, IFN- $\gamma$, IL-6, IL-11, MIP-2, IL-12p70, lymphotactin, TNF- $\alpha$, MCP-5, IL-7, IP-10, IL-10, IL-1 $\alpha$, OSM, SCF, and VEGF-A. The concentrations of the third group were lower, in the femtogram range, including RANTES. Notably, in order to collect the peritoneal exudates, one milliliter of PBS was administered to the peritoneal cavities. Although we cannot accurately evaluate the volumes of original peritoneal fluid prior to PBS administration so far, our observation indicates that the volumes were lower than $0.5 \mathrm{~mL}$. Thus, a 3 -fold dilution before and after PBS administration was assumed in the evaluation of the original concentrations of the mediators in peritoneal exudates.

\subsection{Concentrations of Inflammatory Mediators In Vivo Were} Generally Lower in Plasma than Those in Peritoneal Exudates on Day 1 after Thioglycollate Treatment. As shown in Figure 2 and similar to that observed in the exudates, the production of most mediators, except lymphotactin, reached peak levels in plasma on day 1. IL-7, IL-10, IL-11, and IL-12p70 were not detected in the plasma samples. Because comparisons of mediator concentrations between exudates and plasma would allow the source of the increased concentrations of mediators on day 1 to be identified, we calculated the ratios 

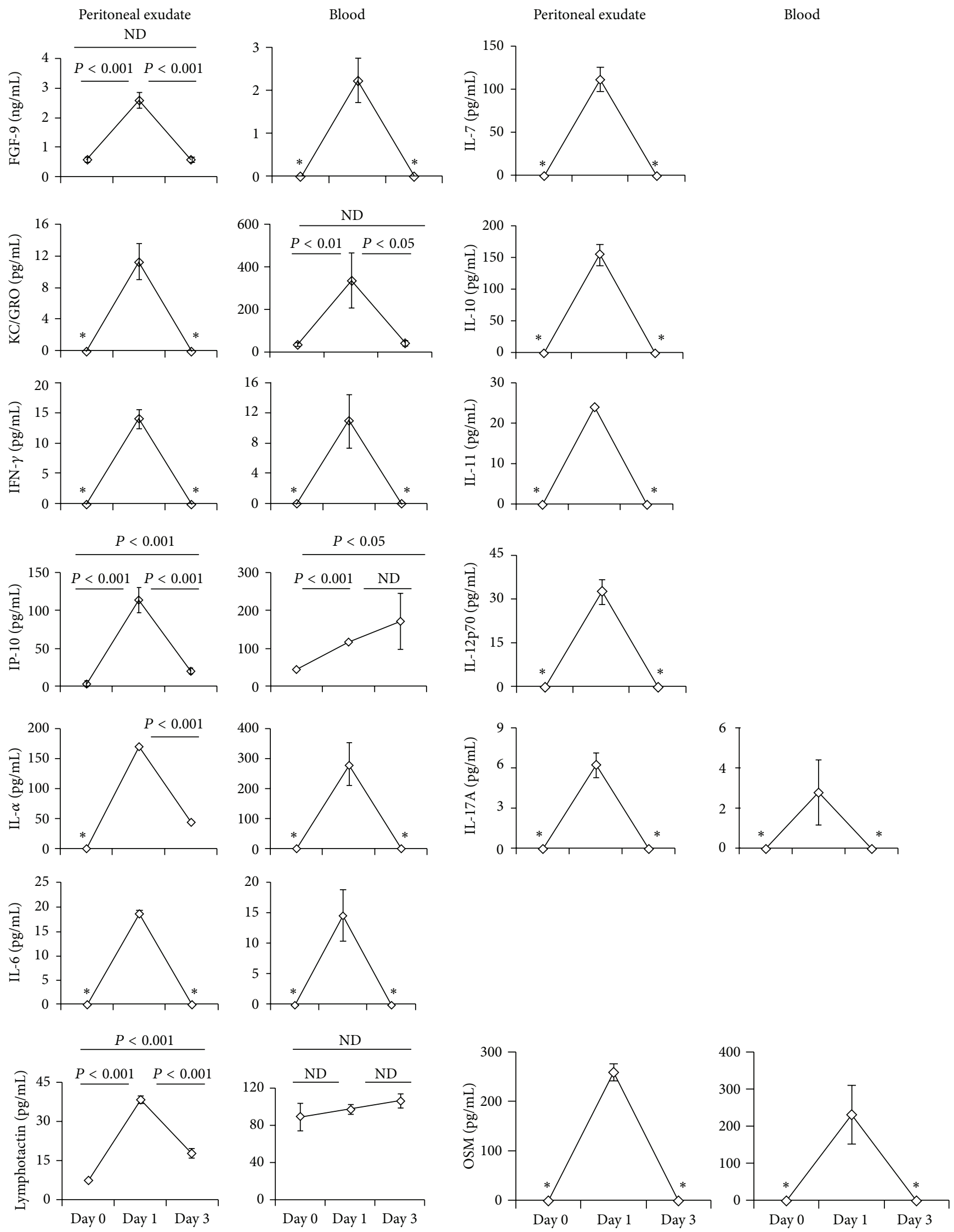

Figure 2: Continued. 

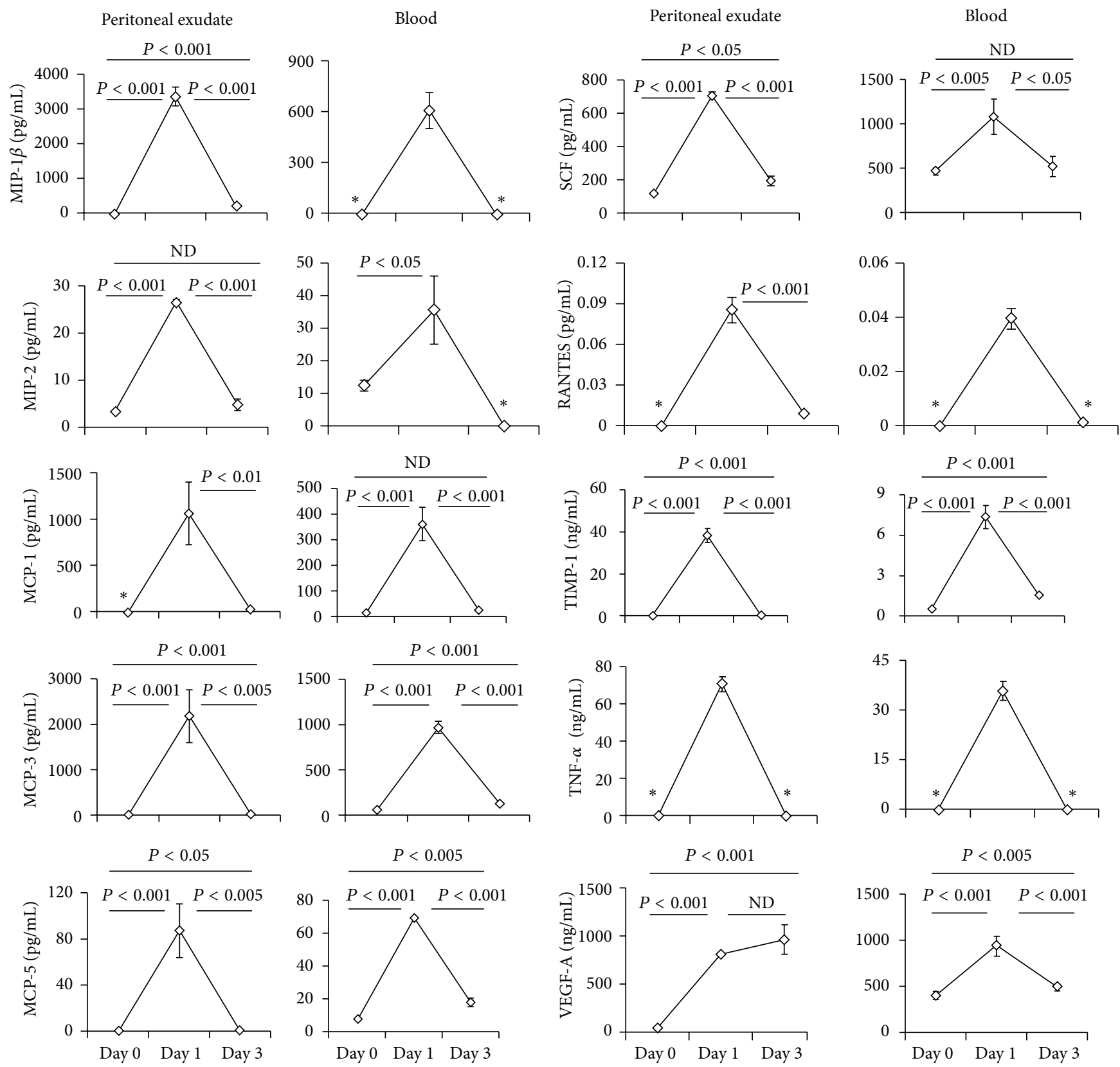

FIGURE 2: Concentration changes of 23 inflammatory mediators in peritoneal exudates and blood during thioglycollate treatment in vivo. C57BL/6 mice (males) were treated with thioglycollate as described in Section 2. At three timepoints, before (day 0) and after (day 1 and 3 ) the treatment, peritoneal exudates and plasma were isolated as described in Section 2 . The concentrations of 23 inflammatory mediators were determined by a bead-based multiplexing immunoassay as described in Section 2. Values are represented as the means \pm SE. $n=3-8$ per timepoint. Data are from two independent experiments. ${ }^{*}$ Denotes that the mediator was undetectable at the indicated timepoint.

of mean values between exudates versus plasma for each mediator concentration. Based on our previous assumption that the exudates were likely diluted 3 -fold, we chose 0.33 as an arbitrary threshold: if the ratio was higher than 0.33 , the concentration of the mediator was likely higher in the exudates than in the plasma, and the source of the increased concentrations of the mediators was likely to be in the exudates. If the ratio was lower than 0.33 , the concentration of the mediator was likely higher in the plasma than in the exudates, and the source of the increased concentrations of the mediators was likely to be in the plasma. There were 19 mediators with detectable concentration levels in both exudates and plasma (Table 1). Among these, 5 mediators exhibited ratios higher than 0.33 in addition to significant differences between the exudate and plasma concentrations $(P<0.05)$; these included lymphotactin, MIP-1 $\beta$, RANTES, 
TABLE 1: Concentrations and ratios of inflammatory mediators in exudates and plasma on day 1 after thioglycollate treatment in vivo*.

\begin{tabular}{|c|c|c|c|}
\hline & $\begin{array}{c}\text { Exudates } \\
(\mathrm{A}, n=3)\end{array}$ & $\begin{array}{c}\text { Plasma } \\
(\mathrm{B}, n=4)\end{array}$ & $\begin{array}{c}\text { Ratio of } \\
\text { mean value } \\
\text { between A } \\
\text { and B }\end{array}$ \\
\hline FGF-9 & $2.60 \pm 0.26$ & $2.23 \pm 0.51$ & 1.17 \\
\hline $\mathrm{KC} / \mathrm{GRO}$ & $11.33 \pm 2.33$ & $335.00 \pm 127.05$ & 0.03 \\
\hline IFN- $\gamma$ & $14.00 \pm 1.53$ & $10.90 \pm 3.52$ & 1.28 \\
\hline IP-10 & $114.00 \pm 16.70$ & $117.50 \pm 5.72$ & 0.97 \\
\hline IL- $1 \alpha$ & $170.33 \pm 3.53$ & $282.00 \pm 71.17$ & 0.60 \\
\hline IL-6 & $18.67 \pm 0.67$ & $14.65 \pm 4.24$ & 1.27 \\
\hline IL-17A & $6.23 \pm 0.92$ & $2.81 \pm 1.62$ & 2.22 \\
\hline Lymphotactin ${ }^{* *}$ & $38.70 \pm 1.45$ & $98.00 \pm 5.28$ & 0.40 \\
\hline MIP-1 $\beta^{* *}$ & $3373.33 \pm 274.73$ & $610.25 \pm 105.45$ & 5.53 \\
\hline MIP-2 & $26.70 \pm 0.67$ & $35.75 \pm 10.38$ & 9.75 \\
\hline MCP-1 & $1069.33 \pm 341.10$ & $364.25 \pm 65.35$ & 2.94 \\
\hline MCP-3 & $2193.33 \pm 579.55$ & $970.50 \pm 64.64$ & 2.26 \\
\hline MCP-5 & $89.00 \pm 21.50$ & $69.25 \pm 1.65$ & 1.29 \\
\hline OSM & $260.00 \pm 17.32$ & $231.25 \pm 79.01$ & 1.12 \\
\hline SCF & $707.00 \pm 21.55$ & $1084.50 \pm 198.52$ & 0.65 \\
\hline RANTES $^{* * *}$ & $0.09 \pm 0.01$ & $0.04 \pm 0.00$ & 2.17 \\
\hline TIMP-1** & $38.67 \pm 3.18$ & $7.40 \pm 0.85$ & 5.23 \\
\hline TNF- $\alpha^{* *}$ & $71.67 \pm 2.96$ & $35.75 \pm 2.93$ & 2.01 \\
\hline VEGF-A & $820.33 \pm 18.44$ & $943.00 \pm 109.18$ & 0.87 \\
\hline \multicolumn{4}{|c|}{$\begin{array}{l}\text { The unit for all concentrations is } \mathrm{pg} / \mathrm{mL} \text {, except FGF- } 9 \text { and TIMP- } 1 \text { as } \\
\mathrm{ng} / \mathrm{mL} \text {. } \\
{ }^{* * *} P<0.001 \text {. } \\
{ }^{* * *} P<0.05 \text {. } \\
\text { All other samples } P>0.05 \text {. }\end{array}$} \\
\hline
\end{tabular}

TIMP-1, and TNF- $\alpha$, indicating their origins from exudates. Thirteen mediators exhibited ratios higher than 0.33 and had no significant differences between exudate and plasma concentrations $(P>0.05)$; these included MCP-1, MCP3, FGF-9, IL-6, MIP-2, IFN- $\gamma$, MCP-5, IP-10, IL- $1 \alpha$, IL$17 \mathrm{~A}, \mathrm{OSM}, \mathrm{SCF}$, and VEGF-A. However, a clear trend was observed in which the concentrations in exudates were higher than those in plasma, indicating that these mediators were highly likely to originate in the exudates. Only one mediator, $\mathrm{KC} / \mathrm{GRO}$, had a ratio lower than 0.33 and an insignificant difference between exudate and plasma concentrations $(P>$ $0.05)$. These findings indicate that, for most of inflammatory mediators, the increases in mediator concentrations on day 1 likely originate from the exudates.

3.3. Inflammatory Mediator Concentrations In Vivo Were Generally Significantly Reduced in Peritoneal Exudates on Day 3 versus Day 1 after Thioglycollate Treatment. As shown in
Figure 2, there were ten mediators for which the concentrations in the exudates were below the detection limits at both day 0 and day 3 ; these included KC/GRO, IL-17A, IFN- $\gamma$, IL-6, IL-11, IL-12p70, TNF- $\alpha$, IL-7, IL-10, and OSM. There were 3 mediators, RANTES, MCP-1, and IL- $1 \alpha$, for which the concentrations in exudates were detectable on day 3 , but not day 0 ; the concentrations of these mediators were significantly reduced on day 3 when compared to those on day $1(P<0.01)$. Another group of 10 mediators were detected at all three timepoints; except for VEGF-A, the concentrations of these mediators were significantly reduced on day 3 compared to day $1(P<0.005)$. Among these 10 mediators, the day 3 concentrations of FGF- 9 and MIP2 reduced to the levels of those on day $0(P>0.05)$. Thus, the concentrations of the mediators (except VEGF-A) in peritoneal exudates on day 3 were significantly reduced compared to those on day 1 .

3.4. Basal Concentrations of Inflammatory Mediators In Vivo Are Higher in Plasma than in Exudates. As shown in Figure 2, there were eight inflammatory mediators that were detected in both plasma and exudates on day 0: IP-10, lymphotactin, MIP-2, MCP-3, MCP-5, SCF, TIMP-1, and VEGF-A. Table 2 compared the concentrations of these detectable mediators and calculated the ratios of mean values between plasma and exudate concentrations. Using an estimated threshold of 0.33 as previously described, the concentrations of these eight mediators were significantly higher in plasma than in exudates at basal conditions $(P<0.001)$.

3.5. LPS Induces the Production of Inflammatory Mediators In Vitro in Peritoneal MФ That Were Isolated from Mice Treated with Thioglycollate for Three Days. In contrast to the maximum numbers of peritoneal $M \Phi$ that were observed on day 3 after thioglycollate treatment (Figure 1), the general production of inflammatory mediators was reduced significantly at this timepoint in both peritoneal exudates and blood (Figure 2). Thus, an intriguing question is whether the peritoneal $\mathrm{M} \Phi$ switch to a subset with an irreversibly low capacity to produce these mediators between days 1 and 3. To address this issue, peritoneal $M \Phi$ were isolated on day 3, purified, and treated with LPS for 24 hours. As shown in Table 3, after the treatment, inflammatory mediator productions were markedly increased in both cell culture media and lysates. There were eleven mediators with greater than 10-fold production increases in culture media; these included IL-1 $\alpha$, MIP-2, MCP-1, MCP-3, IFN- $\gamma$, TIMP-1, TNF$\alpha$, IP-10, MCP-5, IL-6, and MIP-1 $\beta$. Notably, the fold increases in mediator concentrations between culture media and cell lysates were generally consistent $(P>0.05)$, except for IL6, MCP-3, RANTES, and TIMP-1, hinting the participation of posttranslational mechanisms in the regulation of the production of these mediators.

\section{Discussion}

In this study, we investigated the spatiotemporal profiling of multiple inflammatory mediators at different times (days 
TABLE 2: Concentrations of inflammatory mediators in exudates and plasma at basal condition in vivo.

\begin{tabular}{|c|c|c|c|c|}
\hline & Exudates $(\mathrm{A}, n=6)$ & Plasma (B, $n=8)$ & $\begin{array}{c}\text { Ratio of mean value } \\
\text { between } A \text { and } B \text { on day } 0\end{array}$ & Ratio on day 1 \\
\hline IP-10 & $3.05 \pm 0.12$ & $44.00 \pm 4.88$ & 0.07 & 0.97 \\
\hline lymphotactin & $7.57 \pm 0.68$ & $89.50 \pm 15.23$ & 0.09 & 0.40 \\
\hline MIP-2 & $3.63 \pm 0.26$ & $12.59 \pm 1.61$ & 0.29 & 9.75 \\
\hline MCP-3 & $3.42 \pm 0.16$ & $59.50 \pm 2.66$ & 0.06 & 2.26 \\
\hline MCP-5 & $0.73 \pm 0.10$ & $7.83 \pm 1.32$ & 0.09 & 1.29 \\
\hline SCF & $119.00 \pm 6.88$ & $472.00 \pm 39.11$ & 0.25 & 0.65 \\
\hline TIMP-1 & $0.20 \pm 0.02$ & $0.61 \pm 0.05$ & 0.32 & 5.23 \\
\hline VEGF-A & $50.62 \pm 12.06$ & $403.50 \pm 43.57$ & 0.13 & 0.87 \\
\hline
\end{tabular}

(1) $P<0.001$ between $\mathrm{A}$ and $\mathrm{B}$ for all groups.

(2) The unit for all concentrations is $\mathrm{pg} / \mathrm{mL}$, except TIMP-1 as $\mathrm{ng} / \mathrm{mL}$.

(3) The ratio on day 1 is derived from Table 1.

TABLE 3: Fold increases of inflammatory mediator productions in cell culture media and lysates of murine peritoneal macrophages following LPS treatment for $24 \mathrm{~h}$ in vitro.

\begin{tabular}{|c|c|c|}
\hline & \multicolumn{2}{|c|}{ Fold increase } \\
\hline & Culture media (A, $n=2)$ & Cell lysates $(\mathrm{B}, n=4)$ \\
\hline IL-11 & $1.66 \pm 0.03$ & $4.65 \pm 1.30$ \\
\hline OSM & $1.78 \pm 0.10$ & $11.91 \pm 3.55$ \\
\hline IL-7 & $1.90 \pm 0.16$ & $8.75 \pm 2.82$ \\
\hline SCF & $1.94 \pm 0.15$ & $11.86 \pm 3.30$ \\
\hline Lymphotactin & $1.98 \pm 0.22$ & $6.14 \pm 1.61$ \\
\hline FGF-9 & $2.22 \pm 0.23$ & $2.48 \pm 1.10$ \\
\hline IL-10 & $3.29 \pm 0.08$ & $7.71 \pm 2.33$ \\
\hline IL-12p70 & $4.36 \pm 0.93$ & $2.20 \pm 0.96$ \\
\hline VEGF-A & $4.81 \pm 1.36$ & $2.72 \pm 0.53$ \\
\hline KC/GRO & $6.21 \pm 2.87$ & $17.28 \pm 5.00$ \\
\hline RANTES ${ }^{* *}$ & $8.93 \pm 3.00$ & $1.87 \pm 0.55$ \\
\hline IL- $1 \alpha$ & $10.44 \pm 2.36$ & $225.12 \pm 126.41$ \\
\hline MIP-2 & $12.61 \pm 6.43$ & $25.01 \pm 11.05$ \\
\hline MCP-1 & $14.17 \pm 0.80$ & $12.95 \pm 1.56$ \\
\hline MCP- $3^{* *}$ & $17.69 \pm 1.20$ & $11.44 \pm 0.98$ \\
\hline IFN- $\gamma$ & $17.94 \pm 1.23$ & $29.28 \pm 11.75$ \\
\hline TIMP-1 ${ }^{* *}$ & $20.87 \pm 3.31$ & $10.32 \pm 0.97$ \\
\hline TNF- $\alpha$ & $20.93 \pm 8.58$ & $42.44 \pm 17.07$ \\
\hline IP-10 & $44.65 \pm 14.11$ & $28.17 \pm 7.03$ \\
\hline MCP-5 & $47.69 \pm 23.83$ & $3.72 \pm 0.32$ \\
\hline IL-6* & $81.43 \pm 11.59$ & $33.04 \pm 4.43$ \\
\hline MIP-1 $\beta$ & $127.89 \pm 103.35$ & $20.52 \pm 9.87$ \\
\hline
\end{tabular}

0,1 , and 3) and in different locations (peritoneal cavity and blood vessels) during thioglycollate treatment in vivo. Thus, our discussion will focus on two immune properties that were revealed by the investigation of multiple mediators, rather than the biological significance of a specific mediator.

\subsection{Chemotactic Gradient between Blood and Peritoneal} Exudates. Leukocyte accumulation in inflamed tissues is a vital immune response to inflammation, which is guided by chemotactic gradients of a goup of proinflammatory mediators. Indeed, thioglycollate induced peritonitis has been widely used as a model with which to investigate the mechanisms behind the correlation between chemotactic gradients and leukocyte accumulation. Using this model, proteins that are newly identified in the process of leukocyte accumulation include a heterotrimeric $\mathrm{G}_{\mathrm{i}}$-protein $\mathrm{G}_{\alpha \mathrm{i} 2}$ [16], polypeptide N-acetylgalactosamine transferase-1 [17], SLP76 [18], ADAP [18], and sphingosine 1-phosphate receptor 2 [19]. Our current studies provided additional evidence to demonstrate these concepts, in which at basal levels, the mediator concentrations generally in the blood were higher than those in exudates (Table 2). This finding indicates the presence of a reversed chemotactic gradient at basal levels between the blood vessels and peritoneal cavity, which likely plays a critical role in the prevention of $M \Phi$ emigration from circulating peripheral blood. Although only some of the mediators show significant differences between exudates and blood on day 1 following thioglycollate treatment, a trend is obvious (Table 1): the mean value ratios of all detectable mediator concentrations in the exudates versus blood were below 0.33 (down to 0.07 ) on day 0 , while all ratios were above 0.33 (up to 9.75) on day 1 . This finding indicates that although inflammatory mediator concentrations in both exudates and blood were increased one day after thioglycollate treatment, the increased concentrations of most mediators in exudates were higher than in blood. These results elucidate that overlapping chemoattractant gradients exist between the blood and peritoneal exudates on day 1 after thioglycollate treatment, which allows $M \Phi$ migration to reach maximum levels on day 3.

Several models have been proposed previously to evaluate chemotactic gradients, such as the in vitro agarose gel-filled 
tissue culture dish [20] and ex vivo cocultures with mouse cremaster muscle in agarose gel [21]. Our studies provide evidence that thioglycollate induced peritonitis is another model with which to study chemotactic gradients in vivo, such as genetically modified mice.

4.2. Inhibition of Inflammatory Mediator Production in $M \Phi$. We were surprised to discover initially that the productions of nearly all inflammatory mediators in both exudates and blood were reduced on day 3 following thioglycollate treatment, because $M \Phi$ are believed to be major producers for inflammatory mediators and $\mathrm{M} \Phi$ numbers reach peak levels on day 3. However, this result can be interpreted by the previous finding that the phagocytosis of apoptotic neutrophils by $M \Phi$ results in the inhibition of proinflammatory mediator production [22]. Although this previous finding is based on in vitro models, our result hints that this early finding could be applied to in vivo models. Because increased cell death, as characterized by early and late apoptotic cells, can be detected as early as 4 hours after thioglycollate treatment [23], it is highly likely that $M \Phi$ gradually lose the ability to produce inflammatory mediators after taking up apoptotic cells in vivo. However, there are some differences between our in vivo study and the previous in vitro study. For example, previous studies showed that treatment with apoptotic cells during monocyte activation increased the production of anti-inflammatory cytokine IL-10, whereas in our model, IL-10 production was not increased on day 3, hinting that regulation of IL-10 production in vivo is more complicated.

Our studies also showed that the increased neutrophil and $\mathrm{M} \Phi$ accumulation promote inflammatory mediator productions within the first day after thioglycollate treatment, indicating that the level of neutrophil and $M \Phi$ accumulation determines the intensity of inflammation in the early stage of inflammation. The resolution stage of inflammation is defined as the interval from maximum neutrophilic accumulation to the point at which neutrophils are fully removed from the tissue [24]. During this stage, neutrophils are undergoing apoptosis and are subsequently cleared by $M \Phi$ and other phagocytic cells. Our studies also indicated that in the resolution stage of inflammation, increased $M \Phi$ accumulation is not associated with the mediator productions and the level of $M \Phi$ accumulation likely determines the persistence of inflammation.

Extensive evidence from both clinical studies and animal models has implicated the dysregulation of inflammatory mediators as a contributing factor in the pathophysiological progression of cardiovascular disease, such as atherosclerosis [25], abdominal aortic aneurysm, and cardiac infarction [26]. In addition, peritoneal $\mathrm{M} \Phi$ are a reasonable model for mimicking cardiovascular $M \Phi$ responses [27, 28], including foam cell formation [29, 30], MФ recruitment [31, 32], МФ apoptosis [33], and cytokine production [34]. In this regard, the regulation of $М \Phi$ accumulation has been proposed as a therapeutic approach to regulate inflammatory mediator production $[35,36]$. Our results hint that the optimal period of this therapeutic intervention is likely to be in the early stage of inflammation.

\section{Abbreviations}

$\begin{array}{ll}\text { FBS: } & \text { Fetal bovine serum } \\ \text { FGF: } & \text { Fibroblast growth factor } \\ \text { IFN: } & \text { Interferon } \\ \text { IL: } & \text { Interleukin } \\ \text { i.p.: } & \text { Intraperitoneal } \\ \text { IP-10: } & \text { IFN- } \gamma \text {-inducible protein } 10 \\ \text { KC/GRO: } & \text { Growth-regulated } \alpha \text { protein } \\ \text { LDD: } & \text { Least detectable dose } \\ \text { LPS: } & \text { Lipopolysaccharide } \\ \text { MCP: } & \text { Monocyte chemotactic protein } \\ \text { MIP: } & \text { Macrophage inflammatory protein } \\ \text { MФ: } & \text { Macrophages } \\ \text { OSM: } & \text { Oncostatin-M } \\ \text { RANTES: } & \text { T-cell-specific protein } \\ \text { SCF: } & \text { Stem cell factor } \\ \text { SE: } & \text { Standard error } \\ \text { TIMP: } & \text { Tissue inhibitor of metalloproteinases } \\ \text { TNF: } & \text { Tumor necrosis factor } \\ \text { VEGF: } & \text { Vascular endothelial growth factor. }\end{array}$

\section{Acknowledgments}

This work was supported by an AHA National Scientist Development Grant (0835268N). The works of D. Lam were supported by Voelcker Biomedical Research Academy.

\section{References}

[1] E. E. McGrath, H. M. Marriott, A. Lawrie, S. E. Francis, and I. Sabroe, "TNF-related apoptosis-inducing ligand (TRAIL) regulates inflammatory neutrophil apoptosis and enhances resolution of inflammation," Journal of Leukocyte Biology, vol. 90, pp. 855-865, 2011.

[2] H. W. Kim, Q. Chan, S. E. Afton et al., "Human macrophage ATP7A is localized in the trans-Golgi apparatus, controls intracellular copper levels, and mediates macrophage responses to dermal wounds," Inflammation, vol. 35, pp. 167-175, 2012.

[3] F. Broche and J. M. Tellado, "Defense mechanisms of the peritoneal cavity," Current Opinion in Critical Care, vol. 7, no. 2, pp. 105-116, 2001.

[4] J. C. Williams, N. J. Wagner, H. S. Earp, B. J. Vilen, and G. K. Matsushima, "Increased hematopoietic cells in the mertk/- mouse peritoneal cavity: a result of augmented migration," Journal of Immunology, vol. 184, no. 12, pp. 6637-6648, 2010.

[5] K. Moro, T. Yamada, M. Tanabe et al., "Innate production of TH 2 cytokines by adipose tissue-associated c-Kit+ Sca-1+ lymphoid cells," Nature, vol. 463, no. 7280, pp. 540-544, 2010.

[6] N. Topley, Z. Brown, A. Jorres et al., "Human peritoneal mesothelial cells synthesize interleukin-8: synergistic induction by interleukin- $1 \beta$ and tumor necrosis factor- $\alpha$," American Journal of Pathology, vol. 142, no. 6, pp. 1876-1886, 1993.

[7] N. Topley, T. Liberek, A. Davenport, F. K. Li, H. Fear, and J. D. Williams, "Activation of inflammation and leukocyte recruitment into the peritoneal cavity," Kidney International, vol. 50, no. 56, pp. S17-S21, 1996.

[8] M. N. Ajuebor, A. M. Das, L. Virág, R. J. Flower, C. Szabó, and M. Perretti, "Role of resident peritoneal macrophages and mast cells in chemokine production and neutrophil migration in 
acute inflammation: evidence for an inhibitory loop involving endogenous IL-10," Journal of Immunology, vol. 162, no. 3, pp. 1685-1691, 1999.

[9] M. J. Melnicoff, P. K. Horan, and P. S. Morahan, "Kinetics of changes in peritoneal cell populations following acute inflammation," Cellular Immunology, vol. 118, no. 1, pp. 178-191, 1989.

[10] G. J. Bellingan, H. Caldwell, S. E. M. Howie, I. Dransfield, and C. Haslett, "In vivo fate of the inflammatory macrophage during the resolution of inflammation: inflammatory macrophages do not die locally, but emigrate to the draining lymph nodes," Journal of Immunology, vol. 157, no. 6, pp. 2577-2585, 1996.

[11] A. Casrouge, J. Decalf, M. Ahloulay et al., "Evidence for an antagonist form of the chemokine CXCL10 in patients chronically infected with HCV,' Journal of Clinical Investigation, vol. 121, no. 1, pp. 308-317, 2011.

[12] S. B. Joseph, M. N. Bradley, A. Castrillo et al., "LXR-dependent gene expression is important for macrophage survival and the innate immune response," Cell, vol. 119, no. 2, pp. 299-309, 2004.

[13] C. C. J. Zavitz, C. M. T. Bauer, G. J. Gaschler et al., "Dysregulated macrophage-inflammatory protein-2 expression drives illness in bacterial superinfection of influenza," Journal of Immunology, vol. 184, no. 4, pp. 2001-2013, 2010.

[14] H. Blumberg, H. Dinh, E. S. Trueblood et al., "Opposing activities of two novel members of the IL-1 ligand family regulate skin inflammation," Journal of Experimental Medicine, vol. 204, no. 11, pp. 2603-2614, 2007.

[15] J. F. Camargo, M. P. Quinones, S. Mummidi et al., "CCR5 expression levels influence NFAT translocation, IL-2 production, and subsequent signaling events during T lymphocyte activation," Journal of Immunology, vol. 182, no. 1, pp. 171-182, 2009.

[16] K. Wiege, D. D. Le, S. N. Syed, S. R. Ali, and A. Novakovic,

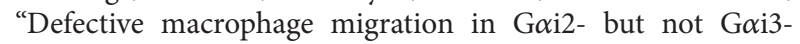
deficient mice," The Journal of Immunology, vol. 189, pp. 980987, 2012.

[17] H. Block, K. Ley, and A. Zarbock, "Severe impairment of leukocyte recruitment in ppGalNAcT-1-deficient mice," The Journal of Immunology, vol. 188, pp. 5674-5681, 2012.

[18] H. Block, J. M. Herter, J. Rossaint, A. Stadtmann, and S. Kliche, "Crucial role of SLP-76 and ADAP for neutrophil recruitment in mouse kidney ischemia-reperfusion injury," The Journal of Experimental Medicine, vol. 209, pp. 407-421, 2012.

[19] J. Michaud, D. S. Im, and T. Hla, "Inhibitory role of sphingosine 1-phosphate receptor 2 in macrophage recruitment during inflammation," Journal of Immunology, vol. 184, no. 3, pp. 14751483, 2010.

[20] E. F. Foxman, J. J. Campbell, and E. C. Butcher, "Multistep navigation and the combinatorial control of leukocyte chemotaxis," Journal of Cell Biology, vol. 139, no. 5, pp. 1349-1360, 1997.

[21] D. C. Cara, J. Kaur, M. Forster, D. M. McCafferty, and P. Kubes, "Role of p38 mitogen-activated protein kinase in chemokine-induced emigration and chemotaxis in vivo," Journal of Immunology, vol. 167, no. 11, pp. 6552-6558, 2001.

[22] V. A. Fadok, D. L. Bratton, A. Konowal, P. W. Freed, J. Y. Westcott, and P. M. Henson, "Macrophages that have ingested apoptotic cells in vitro inhibit proinflammatory cytokine production through autocrine/paracrine mechanisms involving TGF- $\beta$, PGE2, and PAF," Journal of Clinical Investigation, vol. 101, no. 4, pp. 890-898, 1998.

[23] H. Wan, J. M. C. Coppens, C. G. Van Helden-Meeuwsen et al., "Chorionic gonadotropin alleviates thioglycollate-induced peritonitis by affecting macrophage function," Journal of Leukocyte Biology, vol. 86, no. 2, pp. 361-370, 2009.
[24] C. N. Serhan, S. D. Brain, C. D. Buckley et al., "Resolution of inflammation: state of the art, definitions and terms," FASEB Journal, vol. 21, no. 2, pp. 325-332, 2007.

[25] P. J. Little, A. Chait, and A. Bobik, "Cellular and cytokinebased inflammatory processes as novel therapeutic targets for the prevention and treatment of atherosclerosis," Pharmacology and Therapeutics, vol. 131, no. 3, pp. 255-268, 2011.

[26] G. Srinivas, P. Anversa, and W. H. Frishman, "Cytokines and myocardial regeneration: a novel treatment option for acute myocardial infarction," Cardiology in Review, vol. 17, pp. 1-9, 2009.

[27] X. Li, D. Mikhalkova, E. Gao et al., "Myocardial injury after ischemia-reperfusion in mice deficient in Akt2 is associated with increased cardiac macrophage density," American Journal of Physiology, Heart and Circulatory Physiology, vol. 301, pp. H1932-H1940, 2011.

[28] K. Tsujita, K. Kaikita, T. Hayasaki et al., "Targeted deletion of class A macrophage scavenger receptor increases the risk of cardiac rupture after experimental myocardial infarction," Circulation, vol. 115, no. 14, pp. 1904-1911, 2007.

[29] M. C. Levin, P. Jirholt, A. Wramstedt, M. E. Johansson, and A. M. Lundberg, "Rip2 deficiency leads to increased atherosclerosis despite decreased inflammation," Circulation Research, vol. 109, pp. 1210-1218, 2011.

[30] V. P. Yakubenko, A. Bhattacharjee, E. Pluskota, and M. K. Cathcart, " $\alpha \mathrm{m} \beta 2$ integrin activation prevents alternative activation of human and murine macrophages and impedes foam cell formation," Circulation Research, vol. 108, no. 5, pp. 544-554, 2011.

[31] P. Keul, S. Lucke, K. Von Wnuck Lipinski et al., "Sphingosine-1-Phosphate receptor 3 promotes recruitment of monocyte/macrophages in inflammation and atherosclerosis," Circulation Research, vol. 108, no. 3, pp. 314-323, 2011.

[32] T. A. Pagler, M. Wang, M. Mondal et al., "Deletion of ABCA1 and ABCG1 impairs macrophage migration because of increased Racl signaling," Circulation Research, vol. 108, no. 2, pp. 194-200, 2011.

[33] L. Yvan-Charvet, T. A. Pagler, T. A. Seimon et al., "ABCA1 and ABCG1 protect against oxidative stress-induced macrophage apoptosis during efferocytosis," Circulation Research, vol. 106, no. 12, pp. 1861-1869, 2010.

[34] X. Wang, W. Jin, and D. J. Rader, "Upregulation of macrophage endothelial lipase by toll-like receptors 4 and 3 modulates macrophage interleukin-10 and -12 production," Circulation Research, vol. 100, no. 7, pp. 1008-1015, 2007.

[35] C. R. Mackay, "Moving targets: cell migration inhibitors as new anti-inflammatory therapies," Nature Immunology, vol. 9, no. 9, pp. 988-998, 2008.

[36] P. Friedl and B. Weigelin, "Interstitial leukocyte migration and immune function," Nature Immunology, vol. 9, no. 9, pp. 960969, 2008. 


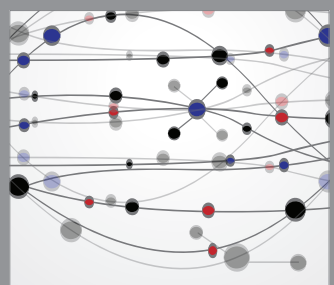

The Scientific World Journal
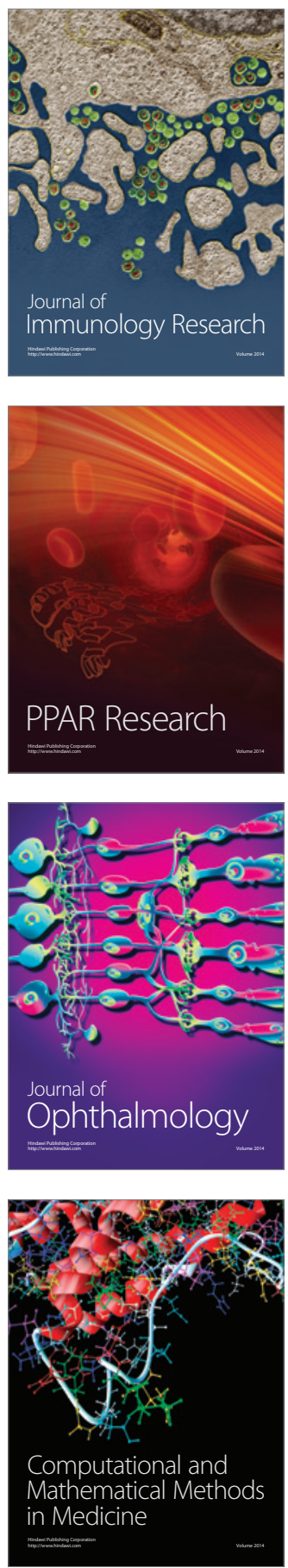

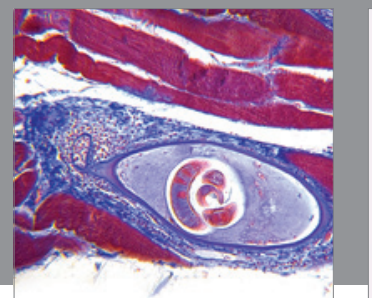

Gastroenterology

Research and Practice
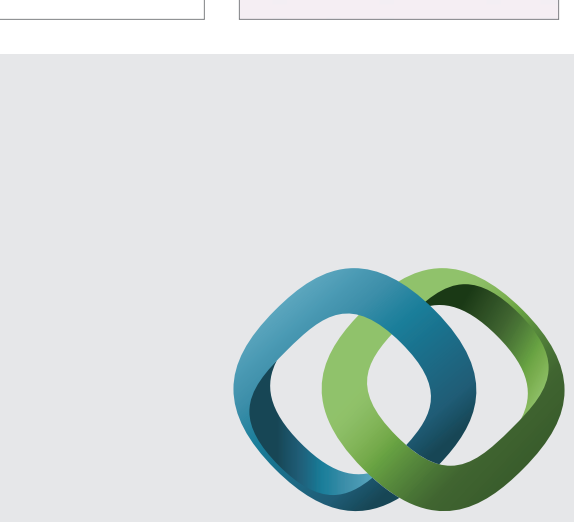

\section{Hindawi}

Submit your manuscripts at

http://www.hindawi.com
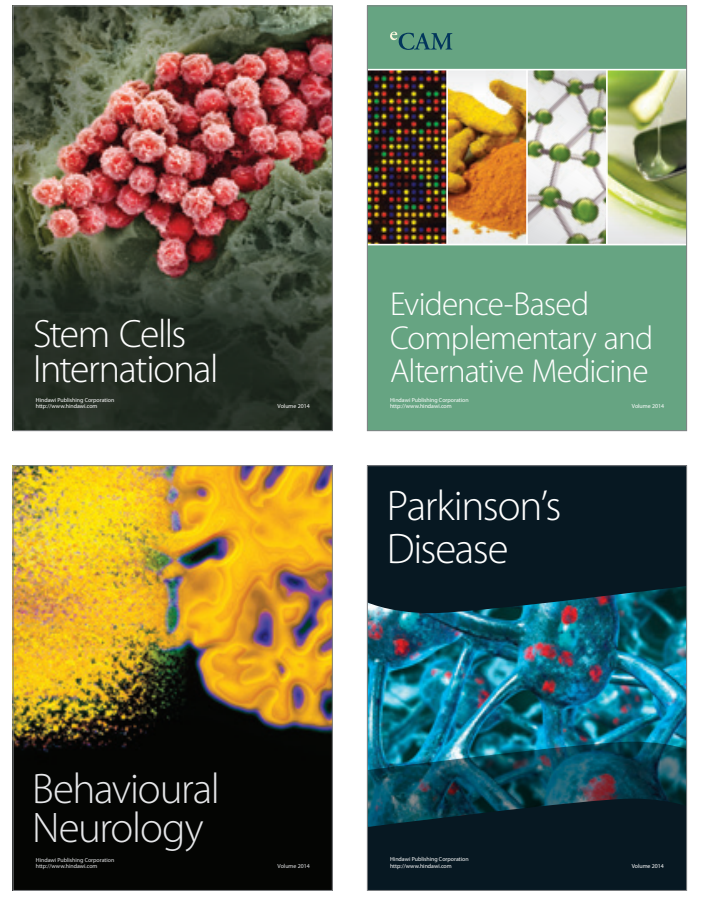
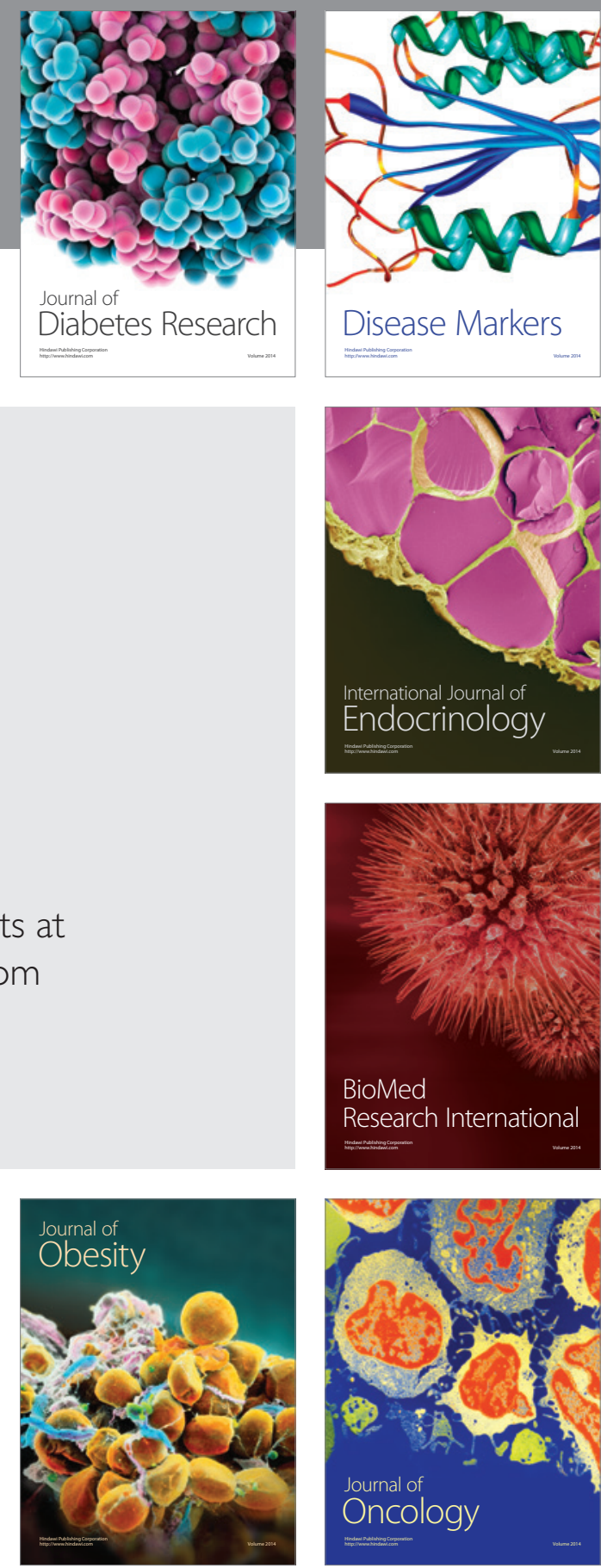

Disease Markers
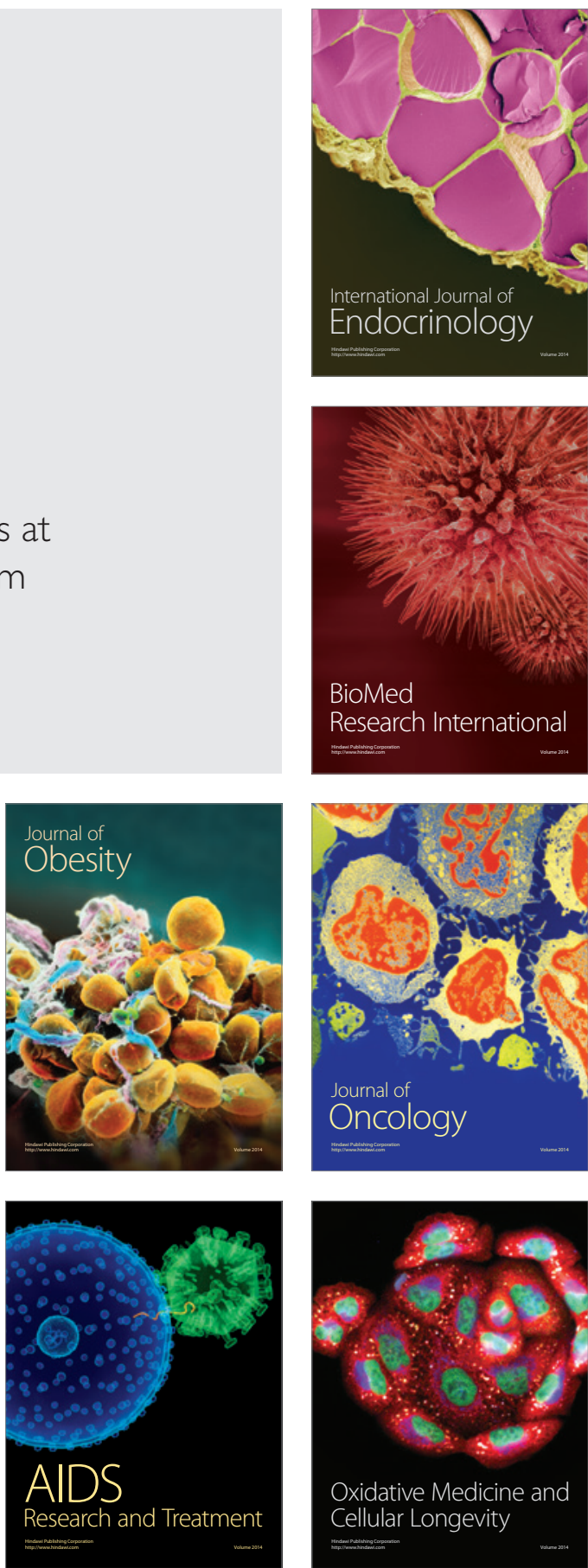